\title{
Oscillating Dynamics and Trajectory of the Single Vortex Line
}

\author{
V. Rusakov ${ }^{a, c}$, S. VAsiliev ${ }^{a, b, *}$, V.V. Chabanenko ${ }^{b}$,

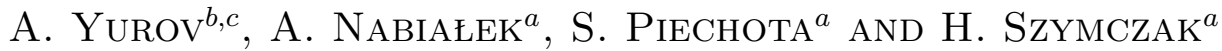 \\ ${ }^{a}$ Institute of Physics, Polish Academy of Sciences \\ al. Lotników 32/46, 02-668 Warsaw, Poland \\ ${ }^{b}$ Donetsk Physico-Technical Institute, Ukrainian Academy of Sciences \\ 72 R. Luxemburg str., 83114, Donetsk, Ukraine \\ ${ }^{c}$ Donetsk National University \\ 24 Universitetskaya str., 83-055, Donetsk, Ukraine
}

The De Gennes and Matricon problem of finding the spectrum of oscillations of massive vortex line under the action of pinning and viscosity forces is solved. The obtained vibration spectrum of the vortex is discussed and the role of each contributing force is elucidated. The trajectory of the vortex motion is calculated and illustrated.

PACS numbers: 74.25.Qt, 74.70.Ad

\section{Introduction}

The problem of vortex dynamics in type-II superconductors is one of the most intensively studied since its discovery by Abrikosov in 1957. Seven years later in 1964 De Gennes and Matricon published an article [1], in which Abrikosov vortex dynamics was considered. In the case of very small induction $B / \Phi_{0} \ll \lambda^{2}$ one can neglect exponentially small interactions between vortices and consider single vortex dynamics [4]. Here by $\Phi_{0}$ we denote the flux quantum and by $\lambda$ the London penetration depth. The authors of Ref. [1] considered two forces acting on a massless vortex, namely line tension $\boldsymbol{F}_{\text {str }}$ and Magnus force $\boldsymbol{F}_{\mathrm{L}}$ (which is very similar to the Lorentz force acting on charged particle, when flux quantum is considered as a charge). In such case the following equation for the single vortex was written:

$$
\boldsymbol{F}_{\mathrm{L}}+\boldsymbol{F}_{\mathrm{str}}=\mathbf{0}
$$

*corresponding author; e-mail: nabia@ifpan.edu.pl 
As a result the quadratic dispersion relation that starts from $\left.\omega(k)\right|_{k=0}=0$ was obtained. This problem was also studied in several other articles [3, 4].

In the present work we consider single Abrikosov vortex line under the action of these forces accompanied by pinning force $\boldsymbol{F}_{\mathrm{p}}$, and viscosity force $\boldsymbol{F}_{\mathrm{V}}$ and additionally we take into account effective mass per unit length of the vortex. The problem changes now significantly, as is seen from dispersion relations and other results presented below.

In these conditions we can describe the motion of the vortex line by a Newton equation $[2,5-9]$ :

$$
\boldsymbol{F}_{\mathrm{L}}+\boldsymbol{F}_{\mathrm{str}}+\boldsymbol{F}_{\mathrm{p}}+\boldsymbol{F}_{\mathrm{V}}=m \frac{\mathrm{d} \boldsymbol{V}}{\mathrm{d} t} .
$$

Now we can find dispersion relations for a vortex motion and trajectory of this motion. It is also interesting to compare these results with the results obtained by De Gennes and Matricon.

In Ref. [1], the line tension $\boldsymbol{F}_{\text {str }}$ and the Magnus force $\boldsymbol{F}_{\mathrm{L}}$ were written in the following form:

$$
\begin{aligned}
& \boldsymbol{F}_{\text {str }}=J \frac{\partial^{2} \boldsymbol{s}}{\partial z^{2}}, \\
& \boldsymbol{F}_{\mathrm{L}}=\left\{\alpha V_{y},-\alpha V_{x}, 0\right\},
\end{aligned}
$$

where $\alpha=m^{*} \Phi_{0} / \mu_{0} \lambda^{2} e, J=\frac{\Phi_{0}^{2}}{4 \pi \mu_{0} \lambda^{2}} \ln \left(\frac{\lambda}{\xi}\right), m^{*}-$ effective electronic mass, $e$ - elementary charge, $\mu_{0}=4 \pi \times 10^{-7} \mathrm{H} / \mathrm{m}, \Phi_{0}$ - flux quantum, $\lambda$ - London penetration depth, and $\xi$ - the coherence length. In our consideration the pinning force is a quasi-elastic restoring force [10], which is proportional to the displacement vector, $s$. The viscosity force is a dissipative force, which is a linear function of the vortex segment velocity $[11,12]$ :

$$
\begin{aligned}
& \boldsymbol{F}_{\mathrm{p}}=-\beta \boldsymbol{s}, \\
& \boldsymbol{F}_{\mathrm{V}}=-\eta \boldsymbol{V}, \quad \eta=\frac{1}{2} B_{\mathrm{c} 2} \sigma .
\end{aligned}
$$

In Eq. (6) $B_{\mathrm{c} 2}$ is the upper critical field and $\sigma$ - the conductivity in normal state. In the case of NbTi alloy we obtain $[10,13] \alpha=3.69 \times 10^{-7} \frac{\mathrm{kg}}{\mathrm{m} \mathrm{s}}, \beta=0.1 \frac{\mathrm{kg}}{\mathrm{m} \mathrm{s}^{2}}$, $\eta=3.56 \times 10^{-7} \frac{\mathrm{kg}}{\mathrm{m} \mathrm{s}}, J=3.85 \times 10^{-11} \frac{\mathrm{kg} \mathrm{m}}{\mathrm{s}^{2}}$ and $m=1 \times 10^{-18} \frac{\mathrm{kg}}{\mathrm{m}}[14-18]$.

\section{Dispersion relations}

Let us now consider a single Abrikosov vortex line with effective mass per unit length $-m$, under the action of the forces, which we discussed above

$$
\left.\begin{array}{l}
J \frac{\partial^{2} s_{x}}{\partial z^{2}}+\alpha \frac{\partial s_{y}}{\partial t}-\eta \frac{\partial s_{x}}{\partial t}-\beta s_{x}=m \frac{\partial^{2} s_{x}}{\partial t^{2}} \\
J \frac{\partial^{2} s_{y}}{\partial z^{2}}-\alpha \frac{\partial s_{x}}{\partial t}-\eta \frac{\partial s_{y}}{\partial t}-\beta s_{y}=m \frac{\partial^{2} s_{y}}{\partial t^{2}}
\end{array}\right\} .
$$

Putting $s_{x}=A \mathrm{e}^{\mathrm{i}(k z+\omega t)}$ and $s_{y}=B \mathrm{e}^{\mathrm{i}(k z+\omega t)}$ into Eq. (7), we obtain the following dispersion relation [19]: 


$$
\left.\begin{array}{l}
\omega_{(1,2)}=\frac{\alpha-\mathrm{i} \eta}{2 m}\left(-1 \pm \sqrt{1+\frac{J k^{2}+\beta}{(\alpha-\mathrm{i} \eta)^{2}}} m\right) \\
\omega_{(3,4)}=\frac{\alpha+\mathrm{i} \eta}{2 m}\left(1 \pm \sqrt{1+\frac{J k^{2}+\beta}{(\alpha+\mathrm{i} \eta)^{2}}} m\right)
\end{array}\right\} .
$$

Taking into account that our considerations are correct for $k \ll 10^{9} \mathrm{~m}^{-1}$ and assuming the above calculated values of $J, m, \alpha, \beta$, and $\eta$, we can expand this expression into a series by a small dimensionless parameter $\left|\frac{J k^{2}+\beta}{(\alpha \pm i \eta)^{2}} m\right| \ll 1$. In this case, we obtain the following two spectrum branches:

$$
\begin{aligned}
\omega_{1} \approx & \frac{\alpha\left(J k^{2}+\beta\right)}{\alpha^{2}+\eta^{2}}-\frac{\left(J k^{2}+\beta\right)^{2} m\left(\alpha^{3}-3 \alpha \eta^{2}\right)}{\left(\alpha^{2}+\eta^{2}\right)^{3}} \\
& +\mathrm{i}\left[\frac{\eta\left(J k^{2}+\beta\right)}{\alpha^{2}+\eta^{2}}-\frac{\left(J k^{2}+\beta\right)^{2} m\left(3 \alpha^{2} \eta-\eta^{3}\right)}{\left(\alpha^{2}+\eta^{2}\right)^{3}}\right], \\
\omega_{2} \approx & \frac{\alpha}{m}+\frac{\alpha\left(J k^{2}+\beta\right)}{\alpha^{2}+\eta^{2}}-\frac{\left(J k^{2}+\beta\right)^{2} m\left(\alpha^{3}-3 \alpha \eta^{2}\right)}{\left(\alpha^{2}+\eta^{2}\right)^{3}} \\
& +\mathrm{i}\left[\frac{\eta}{m}-\frac{\eta\left(J k^{2}+\beta\right)}{\alpha^{2}+\eta^{2}}+\frac{\left(J k^{2}+\beta\right)^{2} m\left(3 \alpha^{2} \eta-\eta^{3}\right)}{\left(\alpha^{2}+\eta^{2}\right)^{3}}\right] .
\end{aligned}
$$

The plots of $\omega_{1}^{\prime}(k)=\operatorname{Re} \omega_{1}(k), \omega_{2}^{\prime}(k)=\operatorname{Re} \omega_{2}(k), \gamma_{1}(k)=\operatorname{Im} \omega_{1}(k)$, and $\gamma_{2}(k)=\operatorname{Im} \omega_{2}(k)$, calculated for different values of $\eta$ and $\beta$, are shown in Fig. 1

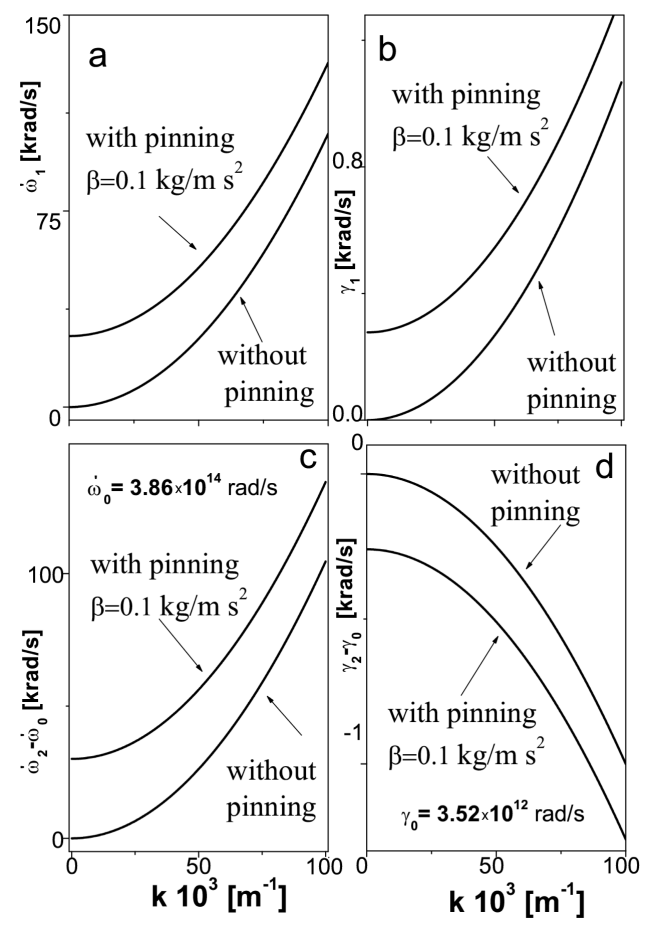

Fig. 1. The influence of the pinning force: (a) on the real part and (b) on the imaginary part of the low frequency oscillation spectrum; (c) on the real part and (d) on the imaginary part of high frequency oscillation spectrum. 

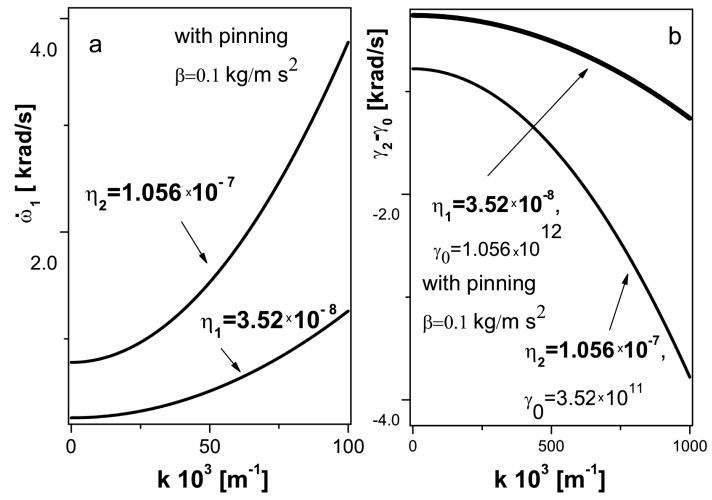

Fig. 2. The influence of the viscosity force (a) on the real part and (b) on the imaginary part of the low frequency branch.

and in Fig. 2. In these figures one can see that the presence of the pinning force leads to the activation type of the spectrum. If we assume all the parameters introduced by us to be equal to zero, the low frequency branch of our spectrum becomes identical to that obtained by De Gennes and Matricon [1].

\section{Trajectory of vortex motion}

Except for studying the dispersion relations, it is also interesting to solve the dynamics' equations of a single vortex. These equations determine displacements of the vortex segments in time as well as a trajectory of the vortex line motion. It is clear that the motion of our vortex is a damped oscillation, which appears either due to initial displacement of the vortex segment from its equilibrium position or due to initial velocity of this segment.

Let us now consider the simplest case $k=0$. The tension of the vortex line, $\boldsymbol{F}_{\text {str }}$, is absent in this case. We can obtain the equations of the vortex motion. However, even in this case we cannot obtain any analytical expression for the vortex trajectory. Nevertheless, we can obtain it numerically.

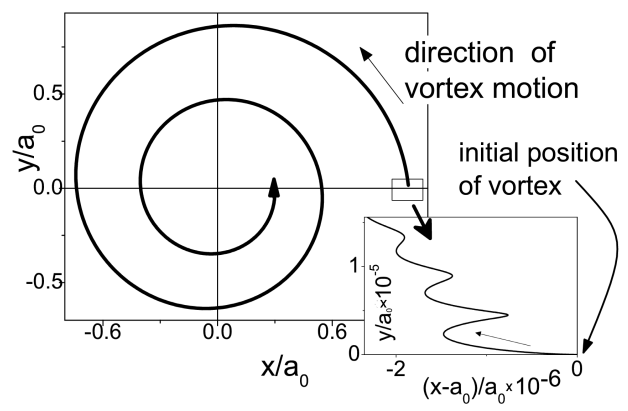

Fig. 3. Trajectory of the vortex line under the influence of the pinning, Lorentz and the viscosity force, for $k=0$. The initial position of the vortex was assumed to be $x=a$ and $y=0$. The initial vortex velocity was assumed to be $V_{x}=V_{y}=0$. 
It is impossible to illustrate the vortex motion in one figure, because there are two different oscillation modes and every oscillation mode is noticeable at its own time interval. The high frequency mode is also very quickly damped $\left(\gamma_{2} \approx 10^{10} \mathrm{~s}^{-1}\right)$. Due to very large differences in frequencies $\left(\omega_{1} \approx 10^{3} \mathrm{~Hz}\right.$ and $\omega_{2} \approx 10^{11} \mathrm{~Hz}$ ) and in amplitudes of these modes, we have imaged the trajectory of the vortex motion in a special picture (see Fig. 3). In the main picture, we illustrated trajectory of vortex motion during $t_{2}=6 \times 10^{-11} \mathrm{~s}$, which is of an order of $2 \pi / \omega_{1}$. In the inset to Fig. 3 we imaged the vortex line motion during $t_{1}=5 \times 10^{-5} \mathrm{~s}$, which is of an order of $2 \pi / \omega_{2}$.

\section{Conclusion}

We have investigated oscillations of a single vortex line, taking into account its dynamical equation, the pinning force, the viscosity forces, and the effective mass of vortex line per unit length. Dispersion relations for such an undulating movement were obtained and analyzed. We have studied the influence of each force on the dispersion relations and on the dynamical characteristics of the vortex motion. The role of the non-zero mass of the vortex was also investigated. It was shown that the insertion of the effective mass into the motion equation leads to an appearance of the second spectrum branch. The trajectory of vortex motion, in the simplest case of $k=0$, was found.

\section{Acknowledgments}

This work was partly supported by the State Committee for Scientific Research (Poland) under contract No. 4 T10B 02325 and by the MSE of Ukraine under project No. M143-2004.

\section{References}

[1] P.G. De Gennes, J. Matricon, Rev. Mod. Phys. 36, 45 (1964).

[2] A. Kasatkin, B. Rosenstein, Phys. Rev. B 60, 14907 (1999).

[3] A.A. Abrikosov, M.P. Kemoklidze, I.M. Khalatnikov, Zh. Eksp. Teor. Fiz. 48, 765 (1965).

[4] A.L. Fetter, P.C. Hohenberg, P. Pincus, Phys. Rev. B 147, 140 (1966).

[5] A. Kasatkin, B. Rosenstein, Physica C 332, 199 (2000).

[6] Tsuneo Ichigochi, Phys. Rev. B 57, 638 (1998).

[7] E.B. Sonin, V.B. Geshkenbein, A. van Otterlo, G. Blatter, Phys. Rev. B 57, 575 (1998).

[8] J.I. Gittelman, B. Rosenblum, J. Appl. Phys. 39, 2617 (1968).

[9] J.I. Gittelman, B. Rosenblum, Phys. Rev. Lett. 16, 734 (1966).

[10] A.M. Campbel, J.E. Ivets, Appl. Phys. 50, 1249 (2001).

[11] Y.B. Kim, C.F. Hempstead, A.R. Strnad, Phys. Rev. B 131, 2486 (1963).

[12] A.R. Strnad, C.F. Hempstead, Y.B. Kim, Phys. Rev. Lett. 13, 26 (1964). 
[13] E.W. Collins, Applied Superconductivity, Metallurgy and Physics of Titanium Alloys, Plenum Press, New York 1986.

[14] G. Baym, E. Chandler, J. Low. Temp. Phys. 50, 57 (1982).

[15] M.W. Coffey, Phys. Rev. B 49, 9774 (1994).

[16] G.E. Volovik, Pis'ma Zh. Eksp. Teor. Fiz. 65, 201 (1997).

[17] N.B. Kopnin, Pis'ma Zh. Eksp. Teor. Fiz. 27, 417 (1978).

[18] H. Suhl, Phys. Rev. Lett. 14, 226 (1965).

[19] V.F. Rusakov, S. Vasiliev, V.V. Chabanenko, A.Yu. Yurov, Visnyk Donetskogo Universyteta, Ser. A 1, 260 (2005). 